\title{
Modified plastic optical fiber coated graphene/polyaniline nanocompositefor ammonia sensing
}

\begin{abstract}
In this paper, a side polished multimode plastic optical fiber gas sensor coated with graphene/polyaniline nanocomposite is developed for ammonia gas sensing application. Graphene/polyaniline nanocomposite is deposited onto side polished plastic optical fiber by drop-casting method. The proposed sensor is exposed to different concentration of ammonia varies from $1 \%$ to $0.25 \%$ at room temperature. Absorbance response of the gas sensor is monitored and recorded using spectrophotometer system. The absorbance increase linearly with the increase in the ammonia concentrations. The response and recovery time are $24 \mathrm{~s}$ and $71.8 \mathrm{~s}$, respectively.
\end{abstract}

Keyword: Side polished plastic optical fiber; Gas sensor; Graphene/polyaniline nanocomposite; Evanescent sensor; Ammonia 